\title{
El papel de las teorías de la comunicación en la construcción del campo académico de la comunicación. Reflexiones desde la historia, la epistemología y la pedagogía
}

\author{
Recibido: 04 de agosto de 2013 \\ Aceptado: 18 de abril de 2014 \\ Publicado: 28 de noviembre de 2014
}

Marta Rizo García

marta.rizo@uacm.edu.mx

Universidad Autónoma de la Ciudad de México

\begin{abstract}
Resumen: A partir de la exposición de algunas formas de organización de las teorías de la comunicación, se busca identificar los fundamentos teóricos que permiten caracterizar como interdisciplinario al campo de la comunicación, sobre todo marcado por los aportes de ciencias sociales como la sociología, la economía y la psicología social, entre otras. Una vez identificados los principales rasgos de la historia del pensamiento comunicacional, se proponen algunos parámetros para actualizar el campo de las teorías de la comunicación, un campo dinámico y cambiante, sobre todo por las propias particularidades del fenómeno comunicativo, sometido a cambios y modificaciones continuas. En última instancia, se plantean algunas reflexiones epistemológicas y pedagógicas que pretenden coadyuvar a la dinamización del campo de las teorías de la comunicación, a partir de ver el papel que juegan en la construcción de los objetos de estudio del campo y en la formación de profesionales de la comunicación.
\end{abstract}

Palabras clave: Comunicación, campo académico, teorías de la comunicación, epistemología, formación, historia del pensamiento comunicacional.

Abstract: From the exhibition of some forms of organization communication theories, this
text looks for to identify the theoretical foundations that they allow to characterize like
interdisciplinary to the field of the communication, mainly marked by the contributions of
social sciences like sociology, the economy and social psychology, among others. Once
identified the main characteristics of the history of the communicational thought, some
parameters set out to update the field of the theories of the communication, a dynamic and
changing field, mainly by the own particularities of the communicative phenomenon, put
under changes and continuous modifications. In last instance, some epistemological and 
pedagogical reflections consider that they try to help to make more dynamics communication theories field, from seeing the paper which they play in the construction of the study objects of the field and in the formation of professionals of the communication.

Key words: Communication, Academic Field, Communication Theories, Epistemology, Education, Communication Thought History.

\section{Presentación. Breve radiografía histórica de las propuestas de organización de las teorías de la comunicación}

La reconstrucción histórica del campo de la comunicación debe tomar en cuenta varios niveles de análisis, entre los cuales destacan, a decir de Fuentes (2003: 28), el social (programas, asociaciones, publicaciones) y el cognoscitivo o intelectual (conceptos, métodos, articulaciones). En estas páginas nos centramos, específicamente, en el segundo nivel de análisis, toda vez que daremos cuenta de algunas de las principales propuestas de organización de las teorías de la comunicación que, sin duda, permiten plantear algunas hipótesis en torno a cómo se ha configurado cognoscitivamente el campo académico de la comunicación, a partir de qué conceptos, enfoques y miradas ha construido conocimiento en torno al fenómeno que le da nombre. Un fenómeno, la comunicación, que, como se verá posteriormente, es particularmente cambiante y dinámico, sobre todo en la actualidad.

A finales de la década de los noventa, Fuentes (1999: 55) afirmaba que "desde mediados de los años ochenta parecen haberse multiplicado en tal medida las posturas y posiciones desde las cuales se puede investigar la comunicación, que el debate es cada vez más difícil, al haber menos referentes comunes". Esta situación se observa aún en la actualidad, donde como veremos, siguen siendo muchos los referentes y posiciones que se plantean como opciones para investigar (tanto teórica como empíricamente) los fenómenos relacionados con la comunicación.

Las llamadas ciencias de la comunicación, a lo largo de su trayectoria histórica, han tenido su principal asiento en las ciencias sociales y en las humanidades, y no tanto así en las ciencias naturales. Es por ello que las principales influencias que se observan en la historia del pensamiento comunicacional provienen de corrientes principalmente sociológicas, tales como el funcionalismo y la teoría crítica, entre otras.

La revisión de las principales formas de organización de las teorías de la comunicación implica, antes que cualquier otra cosa, situar el debate en torno a la especificidad del campo académico de la comunicación. Como afirma Sarale (2008):

"No decimos nada nuevo al enunciar que el campo académico de la comunicación se caracteriza por su indeterminación y su complejidad en cuanto a la definición de su objeto teórico propio y a su constitución disciplinaria. Estas dificultades para algunos tienen 
que ver, tanto con lo polisémico del término comunicación, como por su triple dimensión en tanto proceso, disciplina y profesión”.

El contexto del campo de la comunicación es particular dada la condición enunciada en la cita anterior: la comunicación es un fenómeno sociocultural, es un campo profesional y es, también, un campo académico. Esta triple condición, si bien aporta una riqueza interesante al campo que nos ocupa, no está exenta de problemáticas, sobre todo en lo que a la institucionalización del campo se refiere. A decir de Fuentes (1999: 62):

"El estatuto disciplinario de los estudios sobre la comunicación es, quizá, el tema crucial de debate sobre el pasado, el presente y, sobre todo el futuro de nuestro campo académico. En él confluyen los múltiples y complejos factores históricos que determinan su institucionalización, tanto en el plano cognoscitivo (saberes teóricometodológicos) como en el social (haceres institucionalizados)".

La legitimación del campo académico de la comunicación siempre ha estado en debate y es "un objeto de lucha, tanto al interior como al exterior del propio campo" (Fuentes, 2003: 19). En este contexto, es particularmente importante detenernos a observar cómo se ha configurado cognoscitivamente el campo académico de la comunicación, y una opción posible para realizar tal ejercicio es la revisión de algunas formas de organización de las teorías de la comunicación a lo largo de la trayectoria histórica del campo.

Siguiendo a Levy y Gurevich (1993: 4), "el ansia por descubrir un paradigma universal de la comunicación ha sido sustituida por una cómoda aceptación del pluralismo teórico" (Fuentes, 2003: 22), algo que puede observarse, precisamente, en la dispersión y amplitud de posicionamientos teóricos presentes a lo largo de la historia del pensamiento comunicacional.

Como ya dijimos anteriormente, una forma posible de radiografiar qué ha sucedido con las teorías de la comunicación en el campo académico es la revisión de algunos manuales de teorías de la comunicación. Esta estrategia parte de considerar la relevancia de someter a diálogo los programas educativos y las fuentes bibliográficas. Como apunta Fuentes (2008: 16), “en los libros se encuentran sistematizados los saberes que los estudiantes deberán apropiar y, quizá, 'aplicar' en el ejercicio de una profesión, en la práctica de la investigación, en su ubicación 'ilustrada' y eficaz en el entorno social”. El mismo autor afirma que

"la pregunta sobre 'los libros fundamentales en (y para) el estudio
académico de la comunicación', y su obligada asociación con la
'teoría de la comunicación', admite diversos tipos de respuestas:
algunas descriptivas, otras normativas; pero ninguna de ellas parece
ser no digamos 'definitiva', sino al menos local y provisionalmente
satisfactoria" (Fuentes, 2008: 16).

Por ello, lo que aquí se presenta es sólo una posibilidad de entre otras muchas. Los sesgos en la elección de los manuales siempre estarán presentes, y únicamente presentamos un 
ejercicio de análisis bibliográfico que permite apuntar algunas coordenadas en torno a la constitución "oficial" del campo académico de la comunicación en lo que a su trayectoria teórica se refiere.

El ejercicio se inserta, también, en la lógica de lo trabajado por el estadounidense James Anderson (1996: 5), quien vincula las comunidades disciplinarias de la comunicación y sus respectivas genealogías teóricas con los campos de la escolaridad o enseñanza de la comunicación. El autor afirma que "entramos en la teoría a través de descripciones normalizadas, en libros de texto, de marcos teóricos generalizados, casi siempre dentro de alguna división tópica" (Fuentes, 2008: 17). En la obra de Anderson, se ponen en evidencia

"por una parte, la dispersión (e incluso inconmensurabilidad) de los referentes teóricos en los que se sustenta intelectualmente el campo y, por otra, la incapacidad de sus 'agentes' más destacados para reconocer (y reorientar) los usos y consecuencias prácticas de la bibliografía disponible, especialmente la diseñada para operar como "libros de texto"' (ibídem: 23).

Y en este contexto general, la situación de la investigación de la comunicación en México, “no tiende hacia una 'convergencia' conceptual o a una 'consolidación' disciplinaria, sino hacia lo contrario: a una creciente diversificación de marcos de fundamentación y a una especialización temática que se basa en marcos 'transdisciplinarios' de las ciencias sociales y las humanidades" (ibidem: 51).

A continuación damos cuenta del análisis de algunos manuales de teorías de la comunicación (figura 1), mismo que nos permitirá apuntar algunas hipótesis sobre el papel de las teorías de la comunicación en la configuración cognoscitiva del campo académico de la comunicación:

Figura 1: Enfoques-paradigmas-corrientes-teorías incluidas en los libros analizados.

\begin{tabular}{|c|c|c|}
\hline AUTORES & TEORÍAS INCLUIDAS & TOTAL \\
\hline $\begin{array}{l}\text { Toussaint } \\
(1975)\end{array}$ & Funcionalismo, estructuralismo, marxismo. & 3 \\
\hline Smith (1976) & $\begin{array}{l}\text { Teoría matemática, teoría sociopsicológica, teoría lingüística, sintáctica, semántica, } \\
\text { pragmática. }\end{array}$ & 6 \\
\hline Paoli (1977) & Funcionalismo, estructuralismo, marxismo. & 3 \\
\hline $\begin{array}{l}\text { De Moragas } \\
\quad(1981)\end{array}$ & $\begin{array}{l}\text { El paradigma de Lasswell, Lazarsfeld y los primeros estudios empíricos, } \\
\text { funcionalismo, la psicología de los efectos, Mass Communication Research, los } \\
\text { estudios sobre cultura de masas, el imperialismo, estructuralismo, sociología } \\
\text { crítica de la comunicación de masas, semiótica y comunicación de masas. }\end{array}$ & 10 \\
\hline
\end{tabular}




\begin{tabular}{|c|c|c|}
\hline Corral (1986) & Teoría de la información, estructuralismo, semiología. & 3 \\
\hline $\begin{array}{l}\text { Wolf } \\
(1987)\end{array}$ & $\begin{array}{l}\text { Teoría hipodérmica, corriente de la persuasión, teoría funcionalista de las } \\
\text { comunicaciones de masas, teoría crítica, teoría culturológica, estudios culturales, } \\
\text { teoría de la información, modelo semiótico-textual, agenda-setting, sociología de } \\
\text { los emisores, newsmaking. }\end{array}$ & 13 \\
\hline $\begin{array}{l}\text { Rodrigo } \\
(1989)\end{array}$ & $\begin{array}{l}\text { Modelo de Lasswell, modelo de Shannon, modelo de Schramm, modelo de } \\
\text { Jackobson, modelo de Maletzke, modelo de la sociosemiótica. }\end{array}$ & 6 \\
\hline $\begin{array}{l}\text { McQuail } \\
\text { (1991) }\end{array}$ & $\begin{array}{l}\text { Marxismo, teoría político-económica de los medios de comunicación, la escuela de } \\
\text { Frankfurt y la teoría crítica, teoría hegemónica de los medios, enfoque sociocultural, } \\
\text { enfoques estructural-funcionalistas, contenidos de los medios, audiencias de los } \\
\text { medios, efectos de los medios. }\end{array}$ & 9 \\
\hline Lazar (1996) & Cibernética, antropología, psicología, semiología/estructuralismo. & 4 \\
\hline Miége (1996) & $\begin{array}{l}\text { Modelo cibernético, enfoque empírico-funcionalista, método estructural, } \\
\text { sociología de la cultura de masas, pensamiento crítico, psicología, pensamiento } \\
\text { macluhaniano, economía política crítica de la comunicación, pragmática, } \\
\text { etnografía de la comunicación, etnometodología, sociología de las interacciones } \\
\text { sociales, sociologías de la técnica y de la mediación, la recepción de los mensajes, } \\
\text { las filosofías de la comunicación. }\end{array}$ & 15 \\
\hline $\begin{array}{l}\text { Mattelart y } \\
\text { Mattelart } \\
\text { (1997) }\end{array}$ & $\begin{array}{l}\text { Psicología de las multitudes, escuela de Chicago, Mass Communication Research, } \\
\text { teoría de la información, teoría crítica, estructuralismo, estudios culturales, } \\
\text { economía política, etnometodologías, teoría de la acción comunicativa, etnografía } \\
\text { de las audiencias. }\end{array}$ & 11 \\
\hline $\begin{array}{l}\text { Rodrigo } \\
(2001)\end{array}$ & $\begin{array}{l}\text { Escuela de Palo Alto, interaccionismo simbólico, construccionismo, } \\
\text { etnometodología, funcionalismo, la escuela de Francfort, economía política, } \\
\text { estudios culturales. }\end{array}$ & 8 \\
\hline Torrico (2004) & Difusionista, crítica, culturalista, actual. & 4 \\
\hline $\begin{array}{l}\text { Igartua y } \\
\text { Humanes } \\
(2004)\end{array}$ & $\begin{array}{l}\text { Mass Communication Research, teoría matemática de la información, teoría crítica } \\
\text { de la escuela de Frankfurt, estructuralismo, estudios culturales, economía política } \\
\text { de los medios, funcionalismo sistémico, mediatización de la cultura, teoría de la } \\
\text { acción comunicativa, semiótica de la comunicación de masas, teoría de los efectos, } \\
\text { agenda-setting, análisis del cultivo, usos y gratificaciones, psicología de los medios. }\end{array}$ & 15 \\
\hline Miller (2005) & $\begin{array}{l}\text { Teorías de la organización simbólica, teorías de la producción de mensajes, teorías } \\
\text { del procesamiento de mensajes, teorías del discurso y la interacción, teorías de } \\
\text { la comunicación en el desarrollo de relaciones, teorías de la comunicación } \\
\text { organizacional, teorías de la comunicación en pequeños grupos, teorías del proceso } \\
\text { de los medios y sus efectos, teorías de los medios y la sociedad, teorías de la cultura } \\
\text { y la comunicación. }\end{array}$ & 10 \\
\hline
\end{tabular}




\begin{tabular}{|c|l|l|l|}
\hline \multirow{2}{*}{$\begin{array}{c}\text { West y Turner } \\
(2005)\end{array}$} & $\begin{array}{l}\text { Teoría de la interacción simbólica, gestión coordenada del significado, teoría de } \\
\text { la disonancia cognoscitiva, teoría de los quebrantos de las expectativas, teoría } \\
\text { de la reducción de la incertidumbre, teoría de la penetración social, teoría del } \\
\text { intercambio social, teoría de las dialécticas relacionales, teoría de la gestión de la } \\
\text { teoría de la cultura organizacional, teoría de la información organizacional, retórica, } \\
\text { dramatismo, paradigma narrativo, estudios culturales, análisis del cultivo, teoría } \\
\text { de los usos y gratificaciones, teoría de la espiral del silencio, teoría del medio, } \\
\text { teoría de la negociación cara a cara, teoría del punto de vista, teoría del grupo } \\
\text { enmudecido, teoría de la acomodación de la comunicación. }\end{array}$ & 25 \\
\hline $\begin{array}{c}\text { Marafioti } \\
\text { (2005) }\end{array}$ & $\begin{array}{l}\text { Estructuralismo, semiología, teoría hipodérmica, análisis funcionalista de las } \\
\text { comunicaciones masivas, la perspectiva sistémica de Niklas Luhmann, la escuela } \\
\text { de Frankfurt, estudios culturales, la propuesta de McLuhan, Manuel Castells y la } \\
\text { teoría de la sociedad de la información. }\end{array}$ & 9 \\
\hline $\begin{array}{c}\text { Lozano } \\
(2007)\end{array}$ & $\begin{array}{l}\text { Enfoque crítico de la escuela de Frankfurt, teoría de la aguja hipodérmica, análisis } \\
\text { funcional, el flujo de la comunicación en dos pasos, sociología de la producción } \\
\text { de mensajes, economía política crítica, imperialismo cultural, análisis del cultivo, } \\
\text { establecimiento de agenda, semiótica y estructuralismo, estudios culturales, usos } \\
\text { y gratificaciones. }\end{array}$ & 12 \\
\hline
\end{tabular}

Fuente: Elaboración propia.

Los datos arrojados en este análisis dan cuenta de la dispersión y falta de consenso en torno a los conocimientos teóricos que constituyen el campo de las "teorías de la comunicación”, denominación bajo la cual se encuentran múltiples enfoques, procedentes de matrices disciplinares distintas y que, por ende, ponen énfasis en procesos distintos a partir de conceptos muy diversos. Este primer apunte permite afirmar, entonces, que las clasificaciones de las teorías de la comunicación son muchas y muy diversas.

En las obras revisadas aparecen desde la división tripartita clásica de las teorías de la comunicación en estructuralismo, funcionalismo y marxismo (presente en las obras de Toussaint y Paoli) hasta la enumeración extensísima de "teorías" sobre los diferentes niveles del fenómeno comunicativo que presentan West y Turner, pasando por recuentos más o menos legitimados de teorías de la comunicación que incluyen no sólo a los estudios funcionalistas, sino también a las teorías críticas, las sociologías culturales y las sociologías interpretativas, clasificación presente, con diferentes niveles de profundidad, en obras como las de Smith, Moragas, Wolf, Rodrigo y McQuail, entre otros.

Estos datos, puramente descriptivos, permiten afirmar lo que ya se ha dicho en muchas ocasiones: el campo académico de la comunicación, pese a su grado elevado de institucionalización, no tiene claridad cabal en torno a las teorías que deben aplicarse para analizar los fenómenos comunicativos. Si algo parece estable en esta amalgama de enfoques teóricos es la presencia de la sociología como fuente teórica indiscutiblemente predominante en las llamadas teorías de la comunicación, algo que observamos en la presencia de la teoría funcionalista, la teoría crítica, los estudios culturales, la economía política, las sociologías interpretativas y los aportes de la psico-sociología. Enfoques, 
los anteriores, cuya importancia para el campo de las teorías de la comunicación pocos investigadores de la comunicación pondrían en duda.

En el siguiente apartado, y tomando como partida las ideas vertidas a partir del análisis de los manuales de teorías de la comunicación, situamos el debate en torno a la triple condición de la comunicación como fenómeno (y por lo tanto como objeto de estudio inter y transdisciplinar), como campo profesional y como campo académico. Y nos centramos, específicamente, en la discusión en torno a la naturaleza disciplinaria, interdisciplinaria y/o transdisciplinaria de este campo de conocimiento.

\section{La comunicación, ¿ciencia, campo, disciplina, interdisciplina, transdisciplina?}

La pregunta por la cientificidad de la comunicación no es nueva y ha dado lugar a múltiples posicionamientos, reflexiones y propuestas. Como afirma León (2009), en un ejercicio de análisis de la producción en el marco del Grupo de Teorías y Metodologías de la Investigación en Comunicación de la Asociación Latinoamericana de Investigadores de la Comunicación (ALAIC), "las cuestiones centrales que patentiza el campo académico de la comunicación, y los desafíos que en la actualidad se le presentan a su investigación, tienen que ver fundamentalmente con su estatuto disciplinario".

Según Morin (1994: 14), una disciplina "tiende naturalmente a la autonomía, por la delimitación de sus fronteras, la lengua que ella se constituye, las técnicas que ella está conducida a elaborar o a utilizar, y eventualmente por las teorías que le son propias" (ápud Pereira, 2005: 418). También, para que un conjunto de conocimientos se constituyan como disciplina debe haber claridad y precisión en el objeto de estudio, algo que no sucede con la comunicación, por la propia dispersión y multiplicidad de significados que el propio término "comunicación" connota".

Si el campo de la comunicación es, por naturaleza, un campo inter y transdisciplinar, es, entre otras razones, porque el propio fenómeno comunicativo es susceptible de ser analizado desde múltiples ópticas y enfoques, y como ha quedado claro anteriormente, las matrices teóricas desde las cuales se ha investigado la comunicación a lo largo de la historia no pueden agruparse en un único "modo de ver" adscrito a una única disciplina. Es claro, entonces, que "la comunicación no puede ser encasillada en los márgenes de

\footnotetext{
1. Un ejemplo de la diversidad de significados del término comunicación lo encontramos en la clasificación que sobre el concepto hace Papalini (2002) con base en la taxonomía de Gallino (1995). La autora propone los siguientes modos de concebir a la comunicación: a) como simple transmisión de un estado o propiedad, que puede referirse a objetos inanimados; b) como un comportamiento de un ser viviente que influye sobre otro; $c$ ) como intercambio de valores sociales; $d$ ) como transmisión de información; $e$ ) como el acto de compartir significados socialmente intercambiados; y f) como formación de una unidad social que comparte valores, un determinado modo de vida y un conjunto de reglas.
} 
un compartimento disciplinario estanco" (Torrico, 2006: 3). En otros términos, Múnera (2010: 12) afirma lo siguiente:

"En los debates realizados en el contexto internacional sobre el
estatuto epistemológico de la comunicación para definir si se
trata de una ciencia, de una disciplina, de una interdisciplina, de
una transdisciplina, de un campo de conocimiento, o incluso, si
como prefiere Fernando Andrach (2001), de una 'indisciplina',
la acepción que más fuerza ha tomado es la de campo trans e
interdisciplinar de conocimiento de las ciencias humanas y
sociales".

Entonces, si la comunicación más bien es un campo inter y transdisciplinario, parece poco factible poder hablar de la comunicación como una ciencia, al menos en el sentido clásico del término. Tradicionalmente, para que un conjunto de conocimientos se constituyan como ciencia se requiere la búsqueda de una verdad universal como valor supremo; la verificación empírica o contrastación con el mundo real y natural, y la objetividad. Es obvio que la producción académica que se ha generado a lo largo de la historia del campo académico de la comunicación no cumple con estos requisitos. Por lo anterior, queda claro que la comunicación está más cercana al concepto de campo:

"Es un campo de conocimiento desde el cual se puede comprender, interpretar e intervenir a múltiples niveles los procesos de interacción y significación a través de la creación, circulación y usos de medios y tecnología y de formas simbólicas con multiplicidad de perspectivas: social, cultural, ética, política, estética y económica, entre otras" (Pereira, 2005: 421).

Los fenómenos comunicativos son hechos sociales que pueden y deben interesar a cualquier disciplina relacionada con la sociedad humana y con el comportamiento humano. O dicho de otra forma, "la comunicación es atravesada por todos los órdenes de lo humano, y también ella los atraviesa” (Sierra, 2005: 93). De ahí que la comunicación sea un objeto de estudio compartido por varias miradas disciplinarias y campos de saber. Así, la comunicación, parafraseando a Sánchez Ruiz (1997: 57), no tiene ni ha tenido un campo disciplinar propio, sino un dominio de estudio, más o menos común, alrededor del cual se ha conformado el campo sociocultural. Este dominio, a decir del autor, "ha sido, es y quizá tendrá que seguir siendo una encrucijada inter y transdisciplinaria dentro de las ciencias sociales y humanas". 
Vassallo y Fuentes (2002: 4) aplican el concepto de campo de Pierre Bourdieu ${ }^{2}$ a la comunicación, y sostienen que

“el campo académico de la comunicación está constituido por: un conjunto de instituciones de educación superior destinadas al estudio y a la enseñanza de la comunicación, donde se produce la teoría, la investigación y la formación universitaria de los profesionales de la comunicación. Lo que implica que en ese campo se pueden identificar varios subcampos: el científico, implicado en prácticas de producción del conocimiento: la investigación académica tiene la finalidad de producir conocimiento teórico y aplicado por medio de la construcción de objetos, metodologías y teorías; el educativo, que se define por prácticas de reproducción de ese conocimiento, es decir, mediante la enseñanza universitaria de materias relacionadas con la comunicación, y el profesional, caracterizado por prácticas de aplicación del conocimiento y que promueve vínculos variados con el mercado de trabajo".

Nuevamente aparece la triple condición de la comunicación, ahora asentada a partir del concepto de campo. La comunicación, así entonces, es simultáneamente un campo educativo, profesional y científico o académico, además de ser, antes que cualquier otra cosa, un fenómeno sociocultural que todo ser humano experimenta cotidianamente y sobre el cual pueden afirmarse muchas cosas.

Como decíamos anteriormente, la comunicación no se ha configurado ni como ciencia ni como disciplina. Pero su institucionalización y la cultura académica que existe sobre el fenómeno comunicativo, y que se observa en la trayectoria de los estudios sobre comunicación que tienen ya más de cien años, permiten hablar de un campo académico, sin duda alguna ${ }^{3}$. Veamos ahora si este campo, de naturaleza interdisciplinaria, puede ser considerado una transdisciplina. Como afirma Nicolescu (1999), el término transdisciplina

"concierne como lo indica el prefijo trans, a lo que simultáneamente es entre las disciplinas, a través de las disciplinas y más allá de toda disciplina. Su finalidad es la comprensión del mundo presente, uno de cuyos imperativos es la unidad del conocimiento" (Múnera, 2010: 15).

2. Bourdieu (1997: 48-49) define al campo como "un espacio social estructurado, un campo de fuerzas, hay dominantes y dominados, hay relaciones constantes, permanentes, de desigualdad, que se ejercen al interior de ese espacio que es también un campo de luchas para transformar o conservar este campo de fuerzas". En el campo se construye una visión interpretativa y de apuesta por la construcción de sentido. Y el campo promueve la existencia de objetos, discursos, sujetos, conocimientos y acciones.

3. Otros autores hablan de la comunicación como una "cultura académica" (Torrico, 2006), entendida como un conjunto de premisas que la mayoría de miembros de una determinada comunidad científica, en este caso la de la comunicación, comparten. 
El fenómeno comunicativo es, sin duda, interdisciplinar, pues sobre él (unidad) se han escrito, investigado, reflexionado e interpretado múltiples cuestiones (diversidad). Prueba de ello es, como ya se ha apuntado anteriormente, la multiplicidad de perspectivas desde las cuales se ha abordado el fenómeno. La "pretensión de articulación total" a la que debiera aspirar la transdisciplinariedad parece no estar aún lograda en el campo académico de la comunicación, toda vez que no parece haber el consenso necesario ni siquiera en la definición del propio fenómeno comunicativo objeto de estudio, polisémico donde los haya y aglutinador de una serie de procesos a los que se da un énfasis distinto según sea la mirada desde la cual lo estemos observando. Lo anterior se sitúa en la línea de lo comentado por otros autores como Pineda (2004), para quien

"los objetos de estudio de la comunicación se han construido desde miradas múltiples pero en sus primeras aproximaciones se mantuvieron parcelas y es en los últimos años, especialmente desde los ochenta en adelante, que se busca integrar en una visión más interdisciplinaria con un sentido de mayor totalidad, para poder avanzar en la construcción de un pensamiento comunicacional transdisciplinario todavía no consolidado".

Martín-Barbero (1992) ahonda en lo anterior, al postular que el necesario avance hacia la transdisciplinariedad en los estudios de la comunicación implica "no significa la disolución de sus objetos en los de las disciplinas sociales sino la construcción de las articulaciones -mediaciones e intertextualidades- que hacen su especificidad".

Desde este punto de vista, la comunicación es, entonces, un campo multidisciplinario $\mathrm{y}$, a lo sumo, interdisciplinario. Al menos en estos momentos. El debate, a veces, roza la paradoja, toda vez que, por un lado, toda actividad humana tiene que ver con la comunicación, y por lo tanto, este fenómeno no puede adscribirse a una única perspectiva teórica ni metodológica. Y por el otro, el estudio de la comunicación se apoya de otros campos de conocimiento como la lingüística, la semiótica, la sociología, aunque no se trate de un agregado de discursos teóricos que la conviertan automáticamente en un dominio de estudios interdisciplinar. Así entonces, estamos ante un objeto de estudio que por su propia naturaleza debe interesar a varias disciplinas, pero esta misma condición hace que la consolidación teórica del campo de la comunicación siga sin lograrse.

\section{Las teorías de la comunicación y la constitución cognoscitiva del campo}

El sentido común del campo académico asume que la comunicación es el objeto de estudio de las ciencias de la comunicación, de aquellas aproximaciones teóricas que se han interesado por la comunicación, sobre todo por los medios de difusión masiva, y han generado datos empíricos sobre alguna de las aristas que componen el fenómeno comunicativo. Se aprecia un enfoque primordialmente sociológico en el abordaje de la comunicación desde el campo académico de la comunicación; un enfoque que ve, entonces, a la comunicación desde estructuras conceptuales sobre todo provenientes de matrices conceptuales sociológicas. 
Para que la comunicación, si es que aun no lo tiene, "llegue a encontrar un campo de reflexión propio, dimensionado, que no tenga que recurrir a explicaciones externas, sino que genere sus propios términos explicativos" (Montes, 1983: 15), es necesario alimentar la consolidación teórica del campo, tener consensos mínimos en torno a los conceptos, enfoques y teorías propias de este dominio de conocimiento.

"La existencia de una ciencia de la comunicación es indemostrable
-en términos lógicos- como consecuencia natural de las limitaciones
formales inherentes a cualquier planteamiento teórico de la misma,
al que se le quiera dar un carácter absolutista, en el sentido de caer
en la falacia de que ya se tiene una teoría totalmente acabada"
(Moreno, 2008).

Ciertamente, es difícil hablar de una teoría completamente acabada sobre la comunicación, pues en este campo no es posible buscar fundamentos definitivos y absolutos del conocimiento científico sobre los fenómenos comunicativos. Cualquier teoría que se pretenda, en cualquier área de conocimiento, siempre será insuficiente e inacabada, y por lo tanto, no responderá a todas las interrogantes que se pueden hacer al respecto. "El ideal de una teoría plenamente acabada de la comunicación es una verdadera utopía" (Moreno, 2008).

Pese al crecimiento del campo académico, la comunicación no ha alcanzado la madurez y estabilidad de otras disciplinas científicas. Como afirma Sierra (2005: 88), "las llamadas ciencias de la comunicación son todavía un campo del saber en construcción, a pesar de los años y esfuerzos para constituirse como campo de estudio, afinar sus metodologías de investigación y establecer sus paradigmas teóricos". Y ello se debe, precisamente, a su insuficiente fundamentación teórica, entre otras razones. Además, la teoría de la comunicación debe librarse de dos viejos lastres (Moreno, 2008): la indefinición de su objeto de estudio ("todo es comunicación") y la identificación con la comunicación mediática. Parte del problema de la indefinición de la comunicación como campo científico es producto, también, de que la comunicación surgió primero de la profesión para luego trasladarse problemáticamente al campo de lo académico-científico.

La escasa claridad en la delimitación del objeto de estudio de la comunicación como campo científico puede conducir, a decir de varios autores, a la marginalidad: "Si el campo comunicacional no crea su propio objeto y método, su propia epistemología, estará destinado a la marginación institucional" (Olmedo, 2007: 3). En la misma línea, Torrico (2004) afirma que "la de la comunicación es un área particularmente afectada por ese síndrome de lo light, esa vacuidad, debido en especial al tipo de demanda coyuntural comercial". Para el autor, el objeto de la comunicación es “el proceso social de producción, circulación mediada, intercambio desigual, intelección y uso de significaciones y sentidos culturalmente situados" (ibidem), afirmación que se asemeja a la propuesta por Fuentes Navarro, quien afirma que la investigación en comunicación tiene como objeto de estudio a la producción social de sentido (Fuentes Navarro, 2003 y 2004). 
La investigación en comunicación, así entonces, aborda un objeto empírico propio, pero lo hace desde objetos teóricos emanados de disciplinas diversas. Por lo anterior, se puede afirmar, recuperando a Follari (2000), que

"no hay autonomía de este campo disciplinar, pues su objeto no surge desde la peculiaridad de constitución de un nuevo campo teórico, sino desde la directa necesidad social de explicarse un espacio concreto de funcionamiento de ámbitos de lo real”.

Fuentes ( $c f$. 2006: 4-5), a lo largo de la década de los noventa, desarrolló un modelo para analizar los procesos de estructuración del campo académico de la comunicación en México, que abarcaba tres escalas, a saber: la escala individual (procesos de constitución de los sujetos, procesos de formación/conformación del habitus, y procesos de profesionalización), la escala institucional (procesos de institucionalización social u organización, procesos de institucionalización cognoscitiva y procesos de especialización de la producción científica) y la escala sociocultural (procesos de auto-reproducción, procesos de legitimación social del campo ante el estado y la sociedad civil y procesos de asimilación/acomodación del sentido del campo y de las prácticas en el cambiante entorno sociocultural de la realidad).

En estas páginas nos hemos centrado en el proceso de institucionalización cognoscitiva del campo académico de la comunicación, y hemos apuntado algunos rasgos que permiten ver qué papel han jugado las teorías de la comunicación en dicho proceso. Como se ha podido observar a partir de los datos descriptivos arrojados del análisis de 18 manuales de teorías de la comunicación, no existe un consenso cabal en torno a los enfoques y perspectivas teóricas desde las cuales se deben observar los fenómenos comunicativos.

El campo de la comunicación, a lo largo de su historia, ha legitimado en cierto modo la división tripartita de teorías en funcionalismo, marxismo y estructuralismo, clasificación que desde hace varias décadas parece ya obsoleta, sobre todo por los cambios que se han sucedido en el propio fenómeno comunicativo, que como muchos objetos de conocimiento de gran parte de las ciencias sociales y humanas, evoluciona a un ritmo mucho mayor que el campo científico que cobija las investigaciones en torno al fenómeno objeto de estudio.

Hemos visto, también, que la trayectoria histórica del campo académico de la comunicación permite hablar de campo, mas no de disciplina o ciencia, afirmación que sigue generando debate en la mayoría de foros de investigadores sobre la comunicación, no sólo en México y América Latina, sino también a nivel internacional. Parece claro que hay consenso en torno a que la comunicación se ha configurado como un campo de conocimiento interdisciplinar; pero no ocurre lo mismo con los enfoques teóricos que serían propios del campo comunicativo, que lo harían ser concebido como generador de una "mirada específica" sobre la realidad social.

Lo anterior hace apremiante la tarea de revisar el campo específico de las teorías de la comunicación. Ante la emergencia de nuevos fenómenos comunicativos es menester configurar nuevas miradas y nuevos enfoques teóricos que permitan más y mejores 
lecturas en torno a la comunicación. Y ello, en parte, debe hacerse a partir de la reflexión pedagógica, es decir, preguntándonos cómo podemos innovar el campo de las teorías de la comunicación y su enseñanza. A ello dedicamos el próximo apartado.

\section{La necesidad de innovar el campo de las teorías de la comunicación y su enseñanza}

Es un hecho que la demanda del campo educativo de la comunicación va in crescendo desde hace ya varias décadas. Tampoco puede negarse que ante el vertiginoso crecimiento del número de escuelas y facultades que ofrecen planes de estudio en comunicación han sido muchas las propuestas para re-pensar cómo estamos formando a los comunicadores y comunicólogos. La ambigüedad y dispersión del objeto académico de la comunicación se ha trasladado también a las aulas, lo cual ha generado "una débil identificación social y una concepción de 'presindencia' que golpea sistemáticamente los afanes y aspiraciones de los actores del campo por legitimar su saber y su saber hacer” (Luna, 1994: 180).

El boom de los estudios de comunicación que se dio en los años ochenta en México estuvo marcado por la tendencia a enseñar el manejo técnico de los medios de comunicación colectiva, esto es, el diseño de mensajes para ser difundidos en distintos soportes. El perfil iba, entonces, dirigido a la formación de profesionales de los medios, y en mucha menor medida, a la formación de científicos sociales capaces de analizar, pensar, investigar e intervenir los fenómenos comunicativos entendidos como fenómenos socioculturales sin los cuales es imposible comprender las realidades actuales ${ }^{4}$.

Desde entonces, incluso desde antes, el debate entre la teoría y la práctica está servido y todavía no parece haber total consenso en torno a cómo superar esta tensión. En palabras de Luna (1994: 184)

"el objeto académico quedó así sometido a la tensión entre la
exigencia teórica, vinculada por la vía de lo ideológico con el
plano del fenómeno, y los requerimientos técnicos en el manejo de
los operadores, tensión que, a su vez, dio origen a la bifurcación
'casi irreconciliable' entre la teoría y la práctica".

4. Para ahondar en el asunto de los perfiles de formación de comunicadores y comunicólogos nos parece importante recuperar la clasificación que propone Fuentes Navarro (1996: 139 y ss.), para quien existen, al menos, tres modelos formativos, a saber: el modelo de formación de periodistas, el modelo del comunicador como intelectual y el modelo del comunicador como científico social. La relación entre los perfiles formativos (a los que agregamos el perfil sociocultural del comunicador) y la formación teórica en comunicación la presentamos en un trabajo anterior (Rizo, 2010). 
En esos momentos de debate en torno a la dispersión del objeto académico de la comunicación y sus efectos en la enseñanza de la comunicación en México, Orozco (1992: 41) afirmó que la comunicación adolecía de una "deficiencia congénita", misma que se manifestaba, según el autor, en los siguientes síntomas: la escisión entre técnica y espíritu; la separación entre teoría y práctica y entre enseñanza e investigación; la ambigüedad de la denominación "ciencias de la comunicación", que no ha logrado articulaciones sólidas ni coherentes; y el reduccionismo de asociar la comunicación únicamente con los medios.

Muchos de los autores que han reflexionado sobre las afectaciones que la indefinición del campo académico acarrea en la enseñanza de la comunicación ponen el acento en la dispersión y ambigüedad presente desde las propias formas de nombrar los planes de estudio en comunicación a nivel superior. Caletti (1991: 26) nos ofrece una clara síntesis al respecto:

"Los distintos espacios institucionales de enseñanza e investigación fueron bautizados, de acuerdo a las concepciones políticoepistemológicas de cada tiempo y lugar y una vez superada la etapa de formación periodística, de distintas maneras: como Ciencias de la Información, atadas al intento de formalización tecnocrática con que llega hasta nosotros el desarrollo de la cibernética; como Comunicación Social, recipendiaria de los impactos acumulados de la sociología de la dependencia, de las nociones críticas sobre la industria cultural, de la brusca inclusión de los sectores populares como actores posibles del drama comunicacional y de los primeros contactos fecundos con el instrumental semiológico para el análisis de este drama; y como Ciencias de la Comunicación, tendencialmente vinculada a esa nueva apertura problemática que sucede al agotamiento de los grandes paradigmas omnicomprensivos que despliega la diversidad de sus objetos posibles como dato irremisible de su propia constitución provisional, al tiempo que regresa a las prácticas específicas a buscar nuevas claridades".

En este último apartado se proponen algunos parámetros para actualizar el campo de las teorías de la comunicación, que ha de ser concebido como un campo dinámico y en constante evolución, sobre todo por las propias particularidades del fenómeno comunicativo, hoy en día sometido a cambios y modificaciones continuas. En última instancia, se plantean algunas reflexiones que pretenden coadyuvar a la dinamización del campo de las teorías de la comunicación, a partir de ver qué papel juegan las teorías en la construcción de los objetos de investigación propios del campo y en la formación de los profesionales de la comunicación.

Enumeramos, a continuación, algunos de los retos que presenta la formación de comunicadores y comunicólogos, con énfasis en aspectos de formación teórica: 
- Antes que cualquier otra cosa, es imprescindible tener claridad en torno al concepto de comunicación. A nivel formativo, es necesario dividir el concepto (entendido siempre como fenómeno sociocultural) en sus respectivos niveles de análisis (de la comunicación intrapersonal a la comunicación colectiva, pasando por la comunicación interpersonal, intergrupal e intragrupal), en sus múltiples modalidades (comunicación directa, comunicación mediada, comunicación digital, etc.) y en sus diversas áreas de aplicación (organizacional, política, educativa, para el desarrollo, entre otras).

- Es necesario superar la división clásica tripartita de las teorías de la comunicación, y pese a que no soslayamos la importancia de recuperar (y releer) el trabajo de los autores clásicos y canónicos en el campo de la comunicación, consideramos necesario ampliar las lecturas y estar actualizados permanentemente en materia de discusión teórica sobre la comunicación.

- La organización de las materias teóricas en los planes de estudio trae consigo varias implicaciones que no hay que perder de vista. Al respecto, algunas interrogantes que podemos plantear son las siguientes: ¿es preferible que las materias estén organizadas por enfoque o por nivel del proceso comunicativo?, ¿de qué manera los estudiantes pueden aprehender con mayor solidez la utilidad de las teorías y conceptos que permiten abordar los fenómenos comunicativos?

- Sin duda, es importante trabajar las múltiples lecturas novedosas en torno a los nuevos fenómenos comunicativos (redes sociales, comunicación digital, virtualidad, redes sociales, etc.). Hay aportes novedosos cuya lectura es, hoy, obligada: las teorías de las hipermediaciones y la filosofía de la tecnología, entre otras. El advenimiento de nuevos fenómenos vinculados con la comunicación (recordemos que, como en el caso de la mayoría de ciencias sociales, el objeto de estudio de la comunicación corre a mayor velocidad que las investigaciones académicas que sobre él se desarrollan) hace necesaria la construcción de nuevos conceptos o, al menos, la discusión en torno a la validez de conceptos canónicos en el campo que deben ser superados; un ejemplo de lo anterior es el debate en torno al tránsito de la comunicación de masas a la comunicación posmasiva).

- Por último, pero no por ello menos importante, hay que fortalecer la relación teoríapráctica en la formación de comunicadores y comunicólogos, para que los propios estudiantes (y también los docentes) sean capaces de vincular los conocimientos teóricos con sus haceres profesionales prácticos (sean estos vinculados con el trabajo en los medios, con la investigación académica, la investigación comercial o el trabajo en algún área de comunicación social de cualquier institución).

Así como es apremiante la discusión de cuestiones como las anteriores en la dimensión pedagógica-educativa del campo de la comunicación, no menos importante es seguir alimentando las discusiones teóricas entre académicos. El reconocimiento de lo sucedido a lo largo de la breve pero sustanciosa historia del campo académico de la comunicación 
en lo que a perspectivas teóricas se refiere hace necesario no dar por zanjado el debate en torno a qué referentes teóricos debemos dominar los investigadores de la comunicación y qué conceptos y juicios podemos proponer para investigar de forma rigurosa los nuevos fenómenos comunicativos. Sólo así, el campo académico de la comunicación podrá seguir generando conocimiento riguroso, apegado a las exigencias académicas y, por supuesto, vinculado con su entorno sociocultural de referencia, al que debe orientarse.

\section{Fuentes consultadas}

Anderson, J. (1996). Communication theory. Epistemological foundations. Nueva York, Londres: The Guilford Press.

Bourdieu, P. (1997). Espacio social y campo de poder. Barcelona: Anagrama.

Caletti, S. (1991). "Profesiones, historia y taxonomías. Algunas discriminaciones necesarias”. Diálogos de la comunicación, núm. 31, pp. 25-36.

Corral, M. (1986). La ciencia de la comunicación en México. Origen, desarrollo y situación actual. México D. F.: Trillas.

De Moragas, M.

_(2011). Interpretar la comunicación. Estudios sobre medios en América y Europa. Barcelona: Gedisa.

_(1981). Teorías de la comunicación de masas. Investigaciones sobre medios en América y Europa. Barcelona: Gustavo Gili.

Follari, R. (2000). "Comunicología Latinoamericana: disciplina a la búsqueda de un objeto”. Pensamiento Comunicacional Latinoamericano, vol. 2, núm. 1. Extraída el 18/ IX/2011 desde http://www2.metodista.br/unesco/PCLA/revista5/forum\%205-3.htm

Fuentes Navarro, R.

_(2008). "Bibliografías, biblionomías, bibliometrías: los libros fundamentales en el estudio de la comunicación”. Comunicación y Sociedad, núm. 10, pp. 15-53.

_(2006). "Las dimensiones cognoscitiva y organizacional en la estructuración del campo académico de la comunicación". UNIrevista, vol. 2, núm. 3. Extraída el 2/VI/2011 desde http://www.unirevista.unisinos.br/_pdf/UNIrev_FuentesNavarro.PDF

_(2004). "Del intercambio de mensajes a la producción de sentido: implicaciones de una perspectiva sociocultural en el estudio de la comunicación”. Quórum Académico, Vol. 1, Núm. 1, (enero - junio 2004), pp. 3-22. Maracaibo (Venezuela): Universidad de Zulia. Recuperado el 2 de enero de 2012, de http://www.revistas.luz.edu.ve/index.php/quac/ article/view/1592/1547

_(2003). "La producción social de sentido sobre la producción social de sentido: hacia la construcción de un marco epistemológico para los estudios de la comunicación", pp. 1540. Vassallo de Lopes, M. (org.) Epistemologia da Comunicação. São Paulo: Loyola. 
(1999). "La investigación de la comunicación en América Latina: condiciones y perspectivas para el siglo XXI”. Diálogos de la comunicación, núm. 56, pp. 52-68.

_(1997). "Retos disciplinarios y posdisciplinarios para la investigación de la comunicación”. Comunicación y Sociedad, núm. 31, pp. 215-239.

_(1996). La investigación de la comunicación en México. Sistematización documental 1986-1994. Guadalajara: Universidad de Guadalajara-ITESO.

Galindo, J. (coord.). Comunicación, ciencia e historia. Fuentes cientificas históricas hacia una comunicología posible. Madrid: McGraw-Hill Interamericana.

Gallino, L. (1995). Diccionario de sociología. México D. F.: Siglo XXI.

Igartua, J. y Humanes, M. (2004). Teoría e investigación en comunicación social. Madrid: Síntesis.

Lazar, J. (1996). La ciencia de la comunicación. México D. F.: Publicaciones Cruz.

León Duarte, G. (2009, octubre 19-22). "Estrategias, posiciones y prácticas científicas en la enseñanza y la investigación de la comunicación en América Latina”. Ponencia presentada en el XIII Encuentro Latinoamericano de Facultades de Comunicación Social. La Habana (Cuba), 19-22 de octubre de 2009. Extraída el 18/VII/2011 desde http://www. dialogosfelafacs.net/ descargas/APP1_Mexico\%20-\%20Gustavo\%20Leon.pdf

Levy, M. y Gurevitch, M. (eds.) (1993). "The future of the field - Between fragmentation and cohesion [special issues]". Journal of Communication, vol. 43, núms. 3 y 4, pp. 1-190.

Lozano, J. (2007). Teoría e investigación de la comunicación de masas. México D. F.: Pearson.

Luna Cortés, C. (1994). "El objeto académico en la enseñanza de la comunicación. Siete versiones y un comentario". Estudios sobre las culturas contemporáneas, vol. 6, núm. 1617, pp. 179-207.

Marafioti, R. (2005). Sentidos de la comunicación. Teorías y perspectivas sobre cultura y comunicación. Buenos Aires: Biblos.

Martín Barbero, J. (1992). "Pensar la sociedad desde la comunicación. Un lugar estratégico para el debate de la modernidad". Diálogos de la comunicación, núm. 32. Extraída el 2/X/2011 desde http://www.dialogosfelafacs.net/revista/upload/articulos/ pdf/32JesusMartin.pdf

Martino, L. (2009). "Teorias da comunicação: o estado da arte no universo de língua española”, pp. 38-54. En García Galindo, J.; Vassallo de Lopes, M. y Vera Balanza, M. (coords.). Construir la sociedad de la comunicación. Madrid: Tecnos. 
Mattelart, A. y Mattelart, M. (1997). Historia de las teorías de la comunicación. Barcelona: Paidós.

McQuail, D. (1991). Introducción a la teoría de la comunicación de masas. Barcelona: Paidós.

Miége, B. (1996). El pensamiento comunicacional. México D. F.: UIA.

Miller, K. (2005). Communication Theories. Perspectives, Processes and Contexts. Nueva York: McGraw-Hill.

Montes, E. (1983). "Hacia una fundamentación de la comunicación como ciencia”. Signo y Pensamiento, vol. II, núm. 2, pp. 12-15. Extraída el 2/II/2012 desde http://www.javeriana. edu.co/signoyp/pdf/0201.pdf

Moreno Pérez, A. (2008, febrero 7). “¿Son las ciencias de la comunicación esencialmente incompletas?”. Extraída el 7/II/ 2008 desde http:/www.laflecha.net/canales/ciencia/ articulos/son-las-ciencias-de-la-comunicacion-esencialmente-incompletas

Múnera, P. (2010). "Una aproximación in-disciplinaria a la epistemología de la comunicación”. Encuentros, vol. 8, núm. 15, pp. 11-23. Extraída el 1/X/2011 desde http:// www.uac.edu.co/images/stories/publicaciones/revistas_cientificas/encuentros/volumen-8no-15/art01.pdf

Nicolescu, B. (1999). La transdisciplinariedad. París: Du Rocher.

Olmedo, G. (2007). "Interrogantes acerca del estatuto epistemológico de la comunicación. Acercamiento a caminos propuestos". Ponencia presentada en las XI Jornadas Nacionales de Investigadores de la Comunicación, UNCUYO, Mendoza. Extraída el 10/I/2009 desde http://redcomunicacion.org/memorias/pdf/2007Giolmedo.pdf

Orozco, G. (1992). "De las disciplinas a los saberes. Hacia una reestructuración de la comunicación desde la academia". Comunicación. Estudios venezolanos de comunicación, núm. 87, pp. 39-45. Extraída el 22/XI/2011 desde http://www.gumilla.org.ve/biblioteca/ bases/biblo/texto/COM199487_39-45.pdf

Paoli, J. (1977). Comunicación e información. Perspectivas teóricas. México D. F.: Trillas.

Papalini, V. (2002). "La comunicación: espacio teórico para la ideología contemporánea y su crítica". Ponencia presentada en el VI Congreso Latinoamericano de Investigadores de la Comunicación, Santa Cruz de la Sierra (Bolivia). Documento mimeografiado.

Pereira, J. (2005). "La comunicación: un campo de conocimiento en construcción". Investigación y desarrollo, vol. 13, núm. 2, pp. 412-441. 
Pineda, M. (2004). "La investigación de la comunicación en América Latina: ¿cómo lo hacemos y hacia dónde vamos?". Ponencia presentada en el VII Congreso de ALAIC. Extraída el 11/XI/2011 desde http://www.alaic.net/VII_congreso/gt/gt_17/gt17\%20p16. html

Rizo García, M. (2010). “Formación teórica en Comunicación. La historia del Pensamiento Comunicacional en el plan de estudios de la licenciatura en Comunicación y Cultura de la Universidad Autónoma de la Ciudad de México". Diálogos de la Comunicación, núm. 80. Extraída el 2/XII/2011 desde http://www.dialogosfelafacs.net/revista/articulos-resulta do.php?ed $=80 \& \mathrm{id}=146$

\section{Rodrigo, $\mathrm{M}$.}

_(2001). Teorías de la comunicación. Ámbitos, métodos y perspectivas. Barcelona: UAB. _(1989). Los modelos de la comunicación. Madrid: Tecnos.

Sánchez Ruiz, E. (1997). "Algunos retos para la investigación mexicana de comunicación. Una reflexión personal (en diálogo con Raúl Fuentes)”. Comunicación y Sociedad, núm. 30 .

Sarale, N. (2008). “Comunicación, cultura, estudios culturales... La (in)definición del objeto de estudio de la comunicación y de su estatuto (in/inter/multi/trans/post) disciplinario". Questión, vol. 11, núm. 20. Extraída el 3/X/2011 desde http://www.perio.unlp.edu.ar/ojs/ index.php/ question/article/viewArticle/676

Sierra, L. (2005). "Una aproximación trans e interdisciplinaria del campo de la comunicación”. Conexão - Comunicação e Cultura, vol. 4, núm. 8, pp. 81-100. Extraída el 19/XII/ 2011 desde http://www.ucs.br/etc/revistas/index.php/conexao/article/ viewFile/106/97

Smith, A. (comp.) (1976). Comunicación y cultura. Buenos Aires: Nueva Visión.

Torrico Villanueva, E.

_(2006) "Acercamiento a la comunicación como cultura académica y a sus posiciones teóricas generales". UNIrevista, vol. 1, núm. 3. Extraída el 13/II/2012desde http://www. unire vista.unisinos.br/_pdf/UNIrev_Torrico.PDF

_(2004). Abordajes y periodos de la teoría de la comunicación. Buenos Aires: Norma.

Toussaint, F. (1975). Crítica de la información de masas. México D. F.: Trillas.

Vassallo de Lopes, M. I. (2009). "O estatuto disciplinar da comunicação e o pensamento complexo", pp. 25-37. En García Galindo, J.; Vassallo de Lopes, M. y Vera Balanza, M. (coords.). Construir la sociedad de la comunicación. Madrid: Tecnos.

Vassallo de Lopes, M. y Fuentes Navarro, R. (2002). Comunicación, campo y objeto de estudio. Guadalajara: ITESO. 
West, R. y Turner, L. (2005). Teoría de la comunicación. Análisis y aplicación. Madrid: McGraw-Hill.

Wolf, M. (1987). La investigación de la comunicación de masas. Crítica y perspectivas. Buenos Aires: Paidós. 